\title{
Geographic patterns of diversification: an example with ectoparasitic insects
}

\author{
BORIS R. KRASNOV ${ }^{1,2 *}$, IRINA S. KHOKHLOVA ${ }^{3}$, GEORGY I. SHENBROT ${ }^{1,2}$ and \\ ROBERT POULIN ${ }^{4}$ \\ ${ }^{1}$ Mitrani Department of Desert Ecology, Jacob Blaustein Institutes for Desert Research, Ben-Gurion \\ University of the Negev, Sede Boqer Campus, 84990 Midreshet Ben-Gurion, Israel \\ ${ }^{2}$ Ramon Science Center, PO Box 194, 80600 Mizpe Ramon, Israel \\ ${ }^{3}$ Wyler Department of Dryland Agriculture, French Associates Institute for Agriculture and \\ Biotechnology of Drylands, Jacob Blaustein Institutes for Desert Research, Ben-Gurion University of \\ the Negev, Sede Boqer Campus, 84990 Midreshet Ben-Gurion, Israel \\ ${ }^{4}$ Department of Zoology, University of Otago, PO Box 56, Dunedin 9054, New Zealand
}

Received 1 April 2008; accepted for publication 3 June 2008

\begin{abstract}
On any spatial scale, the species composition of a taxonomic group often departs from a phylogenetically random subset drawn from the pool of species available on a higher scale. Analysis of the uneven representation of related lineages in different assemblages can reveal the action of various forces shaping their diversification. For any assemblage, unequal diversification among lineages can be estimated using diversity skewness, an index of the balance of a phylogenetic tree whose values increase with increasing differences in diversification rates among tree branches. We tested for geographical patterns in the diversity skewness of flea assemblages parasitic on small mammals in 26 distinct geographic localities from the Palaearctic and 15 from the Nearctic. Overall, diversity skewness of the Nearctic flea assemblage was unexpectedly high compared to that of the global flea fauna, whereas that of the Palaearctic did not depart from the expectations of a null model. On a smaller scale, the diversity skewness of local flea assemblages was sometimes lower, sometimes higher, but, in most of the 41 localities, it did not differ significantly from that of random subsets taken from the species pool available on the larger spatial scale (either the world fauna or that of the biogeographical realm, i.e. Palaearctic or Nearctic). More importantly, among Palaearctic assemblages, diversity skewness increased with increasing latitude and/or decreasing mean air temperatures. The different patterns observed in the Palaearctic and Nearctic may be in part due the fact that flea diversification appears to have been more intense in the former than the latter, and to differences between them in relief and glacial history. Temperature-driven speciation rates may well explain the latitudinal gradient in diversity skewness in the Palaearctic. The results illustrate the action of various biogeographical processes in shaping the uneven differentiation of flea lineages on different spatial scales. (C) 2008 The Linnean Society of London, Biological Journal of the Linnean Society, 2008, 95, 807-814.
\end{abstract}

ADDITIONAL KEYWORDS: diversity skewness - latitudinal gradient - mammals - null models - phylogeny - Siphonaptera.

\section{INTRODUCTION}

The unequal diversification of different lineages within a taxon is a universal phenomenon (Dial \& Marzluff, 1989). The result of this inequality is that some lineages are represented in regional or global

*Corresponding author. E-mail: krasnov@bgu.ac.il faunas and floras by many species or genera, whereas other lineages are monotypic. At first glance, the level of biodiversity at the regional or even the global scale should be determined mainly by the occurrence of species belonging to diverse lineages. However, this may not be the case in reality as, for example, in a local assemblage, the number of species belonging to polytypic genera may not necessarily be higher than 
the number of species belonging to monotypic genera. Nevertheless, even in such a hypothetical case, the number of monotypic genera will likely be higher than the number of polytypic genera. This suggests that estimation of biodiversity should take into account not only the mere number of species, but also their phylogenetic relationships.

Although many geographic patterns of biodiversity have been documented (Rosenzweig, 1995; Gaston, 2003), in most cases, these patterns are based on estimates of biodiversity that are based on species richness without taking into account their phylogenetic relationships. The recent development of molecular and analytical tools has lead to the accumulation of phylogenetic data on a great variety of plant and animal taxa. This, in turn, has allowed an assessment of biodiversity from the evolutionary perspective, considering, for example, phylogenetic trees as histories of the diversification of lineages (Mooers \& Heard, 1997). In particular, the search for geographic patterns in the aforementioned unequal diversification among lineages would allow us to elucidate the role of biogeographic processes as causes of variation in speciation and extinction rates and as forces shaping biodiversity.

Unequal diversification among lineages can be estimated using diversity skewness, which essentially measures the level of balance of a phylogenetic tree (Mooers \& Heard, 1997; Heard \& Cox, 2007). If all lineages have similar diversification rate, then the phylogenetic tree is balanced and diversity skewness is low. By contrast, if the diversification success of some lineages is higher than that of other lineages, the resulting tree will be unbalanced and diversity skewness will be high.

The only study of spatial patterns of diversity skewness carried out to date considered the shape of phylogenetic trees of primates at local, regional, and global scales (Heard \& Cox, 2007). In particular, it was found that the Old World and the New World primate faunas differed sharply in their diversity skewness, with the former being unexpectedly highly skewed and the latter less skewed than expected when compared to a 'biogeographic' null model (i.e. a set of species randomly drawn from a source species pool; Heard \& Cox, 2007). This suggests a difference in the diversification histories of Old World and New World primates, which could have arisen for a variety of reasons. It was also found that, although the diversity skewness of both continental and local assemblages differed from null expectations based on the global phylogeny, local assemblages did not differ in their diversity skewnesses from the expectations based on 'continental' phylogenies. In other words, diversity skewness appeared to be affected by continental but not local processes. Finally, a latitudinal gradient of diversity skewness for local assemblages across Africa was found, with relatively high skewness in northern assemblages and relatively low skewness in southern assemblages. The occurrence of this gradient was considered by Heard \& Cox (2007) as evidence for the existence of spatial patterns of diversity skewness at the regional scale despite the lack of local signal in diversity skewness.

Geographic patterns of diversity skewness uncovered in a study of primatesby Heard \& Cox (2007) remain to be validated by studies on other taxa for the sake of confirming the generality of these patterns. Moreover, support for these patterns will be stronger if they hold for a taxon whose phylogenetic position and life history differ sharply from the taxon for which they were initially documented. In particular, the generality of these patterns should be tested for both free-living and parasitic organisms. This is because, for parasites, coevolution with their hosts could well be the main process of diversification as opposed to larger-scale biogeographical processes (Traub, 1980). In the present study, we searched for spatial patterns in diversity skewness among assemblages of fleas (Siphonaptera) parasitic on small mammals in the Palaearctic and Nearctic.

Fleas are typical mammalian parasites (Medvedev \& Krasnov, 2006) most abundant and diverse on small and medium-sized burrowing species. By focusing on taxa parasitic on small mammals, we are therefore likely to capture patterns common to the whole order Siphonaptera. We calculated diversity skewness for flea assemblages on small mammals in 41 distinct geographic localities from the Palaearctic and the Nearctic. Following Heard \& Cox (2007), we asked whether diversity skewness of continental (i.e. all fleas occurring in all assemblages from the respective realm) and local (i.e. fleas occurring in a particular mammalian host assemblage in a given region) flea assemblages differs: (1) from the 'biogeographic' null expectation and (2) between the Palaearctic and the Nearctic. In addition, we asked whether there are host-related (host species richness) as well as geographic (latitudinal), climatic (air temperature and precipitation) or relief (altitude) correlates of flea diversity skewness.

\section{MATERIAL AND METHODS DATA SETS}

Data were obtained from 41 published studies (26 from the Palaearctic and 15 from the Nearctic) that reported the number of flea species found on each small mammal species occurring in a particular locality (see Supporting Information, Table S1). To avoid the confounding effect of sampling effort, we used only 
data based on no less than 130 host individuals examined per locality. To meet the requirement for a diverse local assemblage (Heard \& Cox, 2007), we used only data from the localities where no less than 10 flea species were recorded. The data sets comprised 300 flea species, which represents approximately $15 \%$ of all recognized flea species in the global fauna (Medvedev, 2005). The Palaearctic assemblages included 194 species (22\% of the entire Palaearctic flea fauna; Medvedev, 2005) and the Nearctic assemblages included 114 (38\% of the entire Nearctic flea fauna; Medvedev, 2005).

\section{ANALYTICAL APPROACH}

Heard \& Cox (2007) introduced a technique for the assessment of local diversity skewness and for comparing it with null expectations. In brief, this technique requires a well-resolved global phylogeny for a taxon and presence/absence data for members of this taxon in a number of local assemblages. Then, smaller phylogenetic trees each consisting of the members of a particular assemblage are derived from the global phylogeny. From these 'local' phylogenies, the diversity skewness is calculated for each local assemblage. The next step is to compare the diversity skewness with a null expectation. For the latter, Heard \& Cox (2007) introduced the concept of 'biogeographic' null as opposed to phylogenetic null. The difference between these two null models is as follows. The phylogenetic null compares diversity skewness of a local or regional assemblage with that expected in a monophyletic clade evolving under the equal-rates Markov null model (i.e. when all lineages have equal diversification rates; Cavalli-Sforza \& Edwards, 1967; Harding, 1971; Mooers \& Heard, 1997). By contrast, the 'biogeographic' null compares the diversity skewness of an assemblage with that expected for a set of species randomly drawn from a source species pool (Heard \& Cox, 2007). It is necessary to use the 'biogeographic' rather than the phylogenetic null in studies of spatial patterns of diversity skewness because: (1) the aims of such studies are to test for the effect of local or regional rather than evolutionary processes and (2) it is inappropriate to use the phylogenetic null model if an assemblage does not represent a monophyletic clade. Consequently, the use of the 'biogeographic' null model not only is justified for tests of spatial patterns in diversity skewness, but also it allows the use of a 'global' tree that does not contain the entire set of a clade's members. Indeed, the absence of some members of a clade from a phylogenetic tree of this clade causes the tree to be 'incomplete' and is thus a source of tree-shape bias (Mooers, 1995). However, the use of the 'biogeographic' null avoids this problem because any biases will likely impact the 'local' and 'global' phylogenies in the same way (Heard \& Cox, 2007).

\section{PhYLOGENETIC TREE}

The 'global' phylogenetic tree was based on the only available molecular phylogeny of fleas, namely that recently constructed by Whiting et al. (2008). This tree includes 128 flea species (approximately 6\% of global fauna) belonging to 83 genera (approximately $34 \%$ of the entire number of flea genera). Our data sets included most of these genera, although this was not the case at the species level. Consequently, the positions of the flea taxa that were not represented in the original tree of Whiting et al. (2008) were determined using their morphologically-derived taxonomy (Poulin et al., 2006). The resulting tree was well resolved and contained only a few polytomies.

\section{DATA ANALYSIS}

A variety of measures of tree imbalance are available (Kirkpatrick \& Slatkin, 1993; Mooers \& Heard, 1997; Agapow \& Purvis, 2002; Blum \& François, 2005). Following Heard \& Cox (2007), we measured diversity skewness using Colless' index $I_{\mathrm{c}}$ (Colless, 1982; Agapow \& Purvis, 2002; Blum, François \& Janson, 2006). Tests for significance against the 'biogeographic' null were conducted by comparing the $I_{\mathrm{c}}$ value calculated for a local assemblage composed of a given number of species, with distributions of random $I_{\mathrm{c}}$ values. To obtain the latter, $I_{\mathrm{c}}$ was calculated for each of 10000 assemblages of the same number of species taken at random from either the 'global' or 'continental' phylogeny. Polytomies were dealt with as suggested by Heard \& Cox (2007). We report $I_{\mathrm{c}}$ values calculated as medians of maximal and minimal $I_{\mathrm{c}}$ for local assemblages with phylogenies containing polytomies (see Heard \& Cox, 2007 for details). The reported $P$-values are one-tailed tests in each direction, namely $P_{\mathrm{h}}$ is the probability that diversity skewness is as high or higher than the expected value, whereas $P_{1}$ is the probability that it is as low or lower than the expected value. Local assemblages for which significance tests of $I_{\mathrm{c}}$ values against the 'biogeographic' null produced $P<0.05$ were considered as having significantly higher or lower than expected skewness, whereas assemblages for which significance tests produced $0.05<P<0.1$ were considered as tending toward higher or lower than expected skewness. Calculations were performed using the software SkewMatic, version 2.01 (Heard \& Cox, 2007).

We compared diversity skewness of local assemblages between the Palaearctic and the Nearctic realms using a Mann-Whitney test. To test whether host- and environment-related factors explain spatial 
variation in the diversity skewness of flea assemblages, we calculated for each locality: (1) host species richness and (2) parameters that describe its geographic position (latitude of the center of a sampling area), climate (mean January and mean July temperatures, mean annual precipitation), and relief (mean elevation across a region). These variables where calculated across the sampling area in each locality (see details in Krasnov et al., 2007). Because some environmental variables strongly correlated with each other, we substituted them with the scores of a principal component analysis. Then, we used generalized linear models with a normal distribution and log-link function, searching for the best model using the Akaike's Information Criterion (AIC) separately for each biogeographic realm. Then, we tested the significance of the parameter estimates in each best model using the Wald statistic.

\section{RESULTS}

Values of diversity skewness for the Palaearctic and the Nearctic assemblages as well as for each local flea assemblage are presented in Table 1 . When compared with the 'global' phylogeny, the diversity skewness of both Palaearctic and Nearctic fleas appeared to be relatively low. Nevertheless, skewness of the Nearctic assemblage was unexpectedly high compared to the 'global' phylogeny using the biogeographic null (Table 1).

Diversity skewness across local assemblages was significantly higher in the Nearctic than in the Palaearctic (Mann-Whitney $U=106.5, P<0.02$; Fig. 1). Nevertheless, the skewness of the majority of local assemblages did not differ significantly from that of the assemblages of species drawn randomly from the 'global' pool, except for three Nearctic assemblages

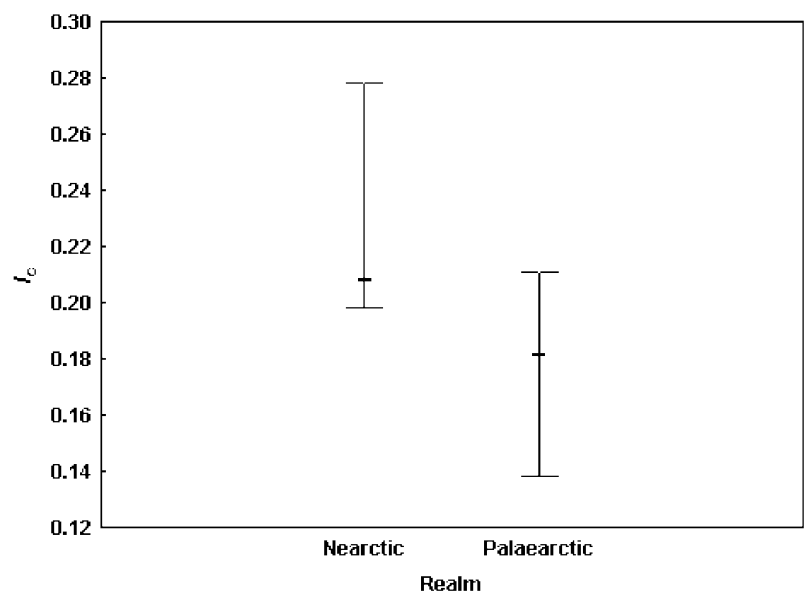

Figure 1. Median and the lower and the upper quartiles of diversity skewness of flea assemblages in both the Nearctic and Palaearctic.
(Montana, New Mexico and Oregon: skewness higher than expected in all) and two Palaearctic assemblages (North-Western Khangay and Northern Russian Asian Far East: skewness higher than expected in both). In addition, the flea assemblage of Eastern Balkhash demonstrated a tendency for its skewness to be lower than expected from the 'global' biogeographic null (Table 1). When compared with the 'realm' phylogenies, the diversity skewness of 15 local assemblages either differed significantly or tended to be different from null expectations. Furthermore, skewness higher than expected was seen in two assemblages only (Texas and Northern Russian Asian Far East), whereas the remaining 13 assemblages demonstrated values of diversity skewness that were either significantly or marginally lower than those of random assemblages (Table 1 ).

The principal component analysis of environmental variables resulted in three factors. These factors explained $93 \%$ of the variance, and their eigenvalues were $2.67,1.31$, and 0.67 . The first factor $\left(F_{1}\right)$ represented a decrease in latitude with a parallel increase in air temperature in January and July (loadings of original variables were $-0.91,0.94$, and 0.75 , respectively), whereas the second factor $\left(F_{2}\right)$ represented an increase in mean altitude (factor loading was 0.97), and the third factor $\left(F_{3}\right)$ represented a decrease in annual precipitation (factor loading was -0.94).

The search for the best model describing the relationships between the diversity skewness of local flea assemblages and host- and environment-related parameters for Nearctic assemblages did not result in any set of independent variables that satisfactorily fitted to the data. The model with the lowest value of AIC (-39.3) involved host species richness as a single explanatory variable, but neither the model nor the respective parameter estimate was significant (likelihood ratio $\chi^{2}=3.46, P=0.06$; Wald statistic $=1.56$, $P=0.21$ ). The best predictor model for the diversity skewness of Palaearctic flea assemblages was the one with both factors $F_{1}$ and $F_{2}$ (AIC $=-71.16$, likelihood ratio $\left.\chi^{2}=13.78, P<0.001\right)$. Moreover, the parameter estimate for factor $F_{1}$ was significant (Wald statistic $=13.65, P<0.001$ ), whereas this was not the case with the parameter estimate for factor $F_{2}$ (Wald statistic $=1.30, P=0.25$ ). The negative value of the parameter estimate for factor $F_{1}(-0.25 \pm 0.07)$ suggests that the diversity skewness of Palaearctic flea assemblages increases with an increase in latitude and/or a decrease of mean summer and winter temperatures (Fig. 2).

\section{DISCUSSION}

This study yielded three main conclusions. First, in flea assemblages, values of diversity skewness signifi- 
Table 1. Diversity skewness $\left(I_{\mathrm{c}}\right)$ of regional flea assemblages and probability that it is higher $\left(P_{\mathrm{h}}\right)$ or lower $\left(P_{\mathrm{l}}\right)$ than that of the reference phylogeny

\begin{tabular}{|c|c|c|c|c|c|}
\hline \multirow[b]{2}{*}{ Region or realm } & \multirow[b]{2}{*}{$I_{\mathrm{c}}$} & \multicolumn{2}{|c|}{$\begin{array}{l}\text { Comparison with the } \\
\text { 'global' phylogeny }\end{array}$} & \multicolumn{2}{|c|}{$\begin{array}{l}\text { Comparison with the } \\
\text { 'realm' phylogeny }\end{array}$} \\
\hline & & $P_{\mathrm{h}}$ & $P_{1}$ & $P_{\mathrm{h}}$ & $P_{1}$ \\
\hline Nearctic & 0.09 & 0.03 & 0.97 & & \\
\hline California central & 0.21 & 0.38 & 0.66 & 0.99 & 0.008 \\
\hline California southwestern & 0.21 & 0.56 & 0.48 & 0.66 & 0.39 \\
\hline Colorado & 0.20 & 0.41 & 0.62 & 0.61 & 0.44 \\
\hline Connecticut & 0.27 & 0.38 & 0.67 & 0.93 & 0.09 \\
\hline Florida & 0.23 & 0.46 & 0.59 & 0.96 & 0.05 \\
\hline Idaho & 0.20 & 0.17 & 0.85 & 0.82 & 0.21 \\
\hline Missouri & 0.19 & 0.88 & 0.24 & 0.38 & 0.68 \\
\hline Montana & 0.41 & 0.03 & 0.98 & 0.94 & 0.08 \\
\hline New Mexico & 0.21 & 0.04 & 0.97 & 0.80 & 0.26 \\
\hline Oregon & 0.33 & 0.04 & 0.97 & 0.63 & 0.41 \\
\hline Tennessee & 0.17 & 0.67 & 0.36 & 0.14 & 0.88 \\
\hline Texas & 0.31 & 0.47 & 0.61 & 0.07 & 0.94 \\
\hline Washington & 0.28 & 0.62 & 0.53 & 0.55 & 0.54 \\
\hline Wisconsin & 0.20 & 0.77 & 0.28 & 0.84 & 0.20 \\
\hline Wyoming & 0.27 & 0.48 & 0.59 & 0.59 & 0.51 \\
\hline Palaearctic & 0.06 & 0.66 & 0.32 & & \\
\hline Adzharia & 0.21 & 0.42 & 0.62 & 0.60 & 0.45 \\
\hline Akmolinsk & 0.14 & 0.83 & 0.19 & 0.95 & 0.06 \\
\hline Dzhungarskyi Alatau & 0.14 & 0.86 & 0.17 & 0.95 & 0.07 \\
\hline East Balkhash & 0.11 & 0.93 & 0.08 & 0.99 & 0.008 \\
\hline Kabarda & 0.20 & 0.46 & 0.58 & 0.65 & 0.39 \\
\hline Krasnojarsk & 0.21 & 0.42 & 0.62 & 0.60 & 0.45 \\
\hline Kustanai & 0.16 & 0.83 & 0.21 & 0.92 & 0.10 \\
\hline Kyrgyz ridge & 0.13 & 0.77 & 0.25 & 0.95 & 0.06 \\
\hline Mongolia, central Khangay & 0.21 & 0.42 & 0.62 & 0.61 & 0.43 \\
\hline Mongolia, northwestern Khangay & 0.18 & 0.02 & 0.98 & 0.12 & 0.89 \\
\hline Morocco & 0.14 & 0.85 & 0.18 & 0.95 & 0.06 \\
\hline Moscow & 0.18 & 0.69 & 0.36 & 0.83 & 0.22 \\
\hline Moyynkum & 0.17 & 0.32 & 0.70 & 0.63 & 0.40 \\
\hline Northern Russian Asian Far East & 0.42 & 0.02 & 0.98 & 0.03 & 0.97 \\
\hline Norway & 0.17 & 0.90 & 0.17 & 0.94 & 0.10 \\
\hline Novosibirsk & 0.14 & 0.72 & 0.31 & 0.93 & 0.09 \\
\hline Pavlodar & 0.30 & 0.29 & 0.75 & 0.37 & 0.68 \\
\hline Pre-Polar Ural & 0.22 & 0.76 & 0.35 & 0.83 & 0.28 \\
\hline Selenga & 0.27 & 0.44 & 0.64 & 0.54 & 0.56 \\
\hline Slovakia & 0.15 & 0.83 & 0.20 & 0.93 & 0.08 \\
\hline Southwestern Azerbaijan & 0.21 & 0.32 & 0.71 & 0.51 & 0.52 \\
\hline Sweden & 0.20 & 0.77 & 0.28 & 0.84 & 0.20 \\
\hline Taimyr & 0.36 & 0.28 & 0.76 & 0.34 & 0.70 \\
\hline Tarbagatai & 0.13 & 0.75 & 0.27 & 0.95 & 0.06 \\
\hline Ural River & 0.21 & 0.71 & 0.37 & 0.80 & 0.26 \\
\hline Western Sayan & 0.14 & 0.79 & 0.23 & 0.95 & 0.06 \\
\hline
\end{tabular}

cantly different from null expectations occurred at both local and continental scales. Second, there is evidence for spatial and/or environmental patterns in diversity skewness. Third, the level of skewness as well as the manifestation of spatial/environmental patterns in its magnitude differed between the two biogeographic realms investigated.

The only other study of spatial patterns in diversity skewness (Heard \& Cox, 2007) found that significant skewness in primate assemblages occurs mainly at 


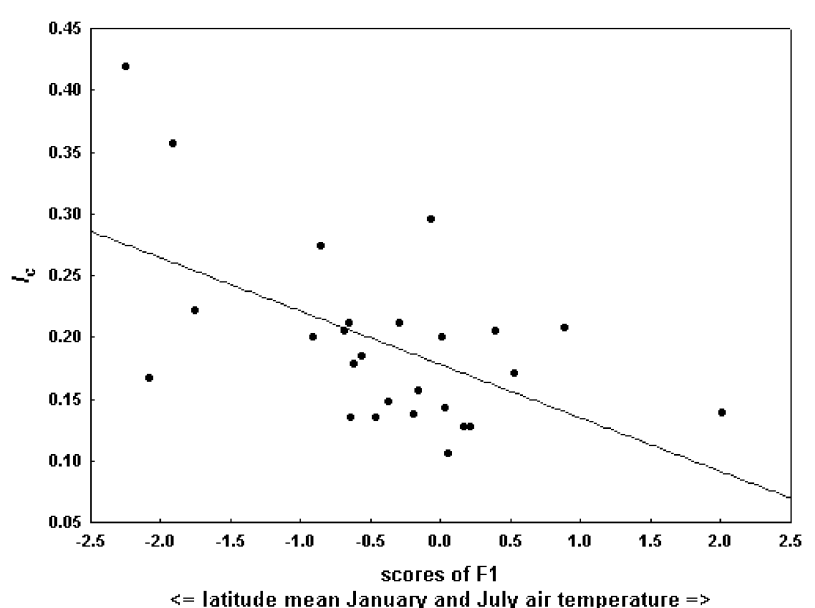

Figure 2. Relationship between the diversity skewness and a composite variable $F_{1}$ (for explanations, see text) across 26 Palaearctic flea assemblages.

the continental rather than at the local scale. In the present study, signicant deviations of skewness from null expectations were found in one of the two realm assemblages and in 15 of 41 local assemblages. The entire Nearctic flea assemblage had unexpectedly high diversity skewness compared to that of random assemblages drawn from the global species pool. Moreover, the local flea assemblages in the Nearctic are characterized by generally higher skewness compared to Palaearctic assemblages. This means that some lineages in the entire Nearctic assemblage as well as in local flea assemblages are represented by disproportionately higher number of species than other lineages.

The numerical dominance of one or very few lineages within a taxon is not uncommon, and could result from grossly unequal rates of speciation and extinction among lineages in a geographical area because of intrinsic differences in their ecological characteristics (Dial \& Marzluff, 1989; Slowinski \& Guyer, 1989, 1993; Barraclough, Vogler \& Harvey, 1998). A related reason for this could be geographically-constrained diversification success in some lineages, which, in turn, could be historically or ecologically based. For example, scincid lizards are represented by an unusually high number of species in New Zealand, resulting presumably from either rapid allopatric speciation during the Oligocene (when New Zealand was fragmented into many islands) or the absence of small mammalian competitors and predators, or both (Hickson, Slack \& Lockhart, 2000). The parasitic lifestyle of all flea species as well as the highly conservative life cycle among all Siphonaptera suggest that ecological explanations for the difference between realms in the level of diversity skewness are unlikely. Certain historical aspects of flea zoogeography may instead provide a more reasonable explanation. Although the geography of flea evolution is debatable (Traub, 1980 versus Medvedev, 2005), it is commonly accepted that the diversification of the majority of flea lineages belonging to large families took place in the Old World, whereas the diversification of the youngest of the large flea families, Ceratophyllidae, likely occurred in North America (Traub, 1980). As a result, the latter is represented in the Nearctic by a disproportionately high number of species (119 of 299 species; Medvedev, $2000 \mathrm{a}, \mathrm{b})$.

The skewness of the majority of local assemblages differed from that expected if species were taken at random from the total species pool of the realm, but did not differ from that expected if species were taken at random from the global species pool. This suggests that the composition of a flea assemblage in a locality arises due to some processes acting on an ecological rather than on an evolutionary scale. In other words, processes such as a differential rate of diversification among flea lineages appear to play a minor (if any) role, whereas the role of processes involving species interactions could be substantial. Furthermore, the general direction of the diversity skewness of local assemblages indicates that it is usually lower than expected. This suggests that different flea lineages are represented in a local flea assemblage in a more balanced way than they are represented in the species pool of the whole realm. In other words, both more diversified and less diversified lineages contribute equally to a local flea assemblage. This may happen if members of highly diversified and poorly diversified lineages differ in their geographic range size. If the latter have generally broader distributions than the former, the probability that they will co-occur in the same locality should obviously be high. However, the relationship between geographic range size and diversification rate is unclear (Rosenzweig, 1995; Chown, 1997; Gaston, 1998). Nevertheless, the observed pattern for parasites may be explained by combining hypothetical relationships between diversification rate, host specificity, and geographic range. Indeed, host-specific parasites are thought to have a higher likelihood of undergoing diversification than host-opportunistic parasites (Poulin \& Morand, 2004). This may lead to a generally higher degree of host specificity among members of a highly diversified lineage. Given that geographic range size in fleas has been shown to correlate negatively with their degree of host specificity (Krasnov et al., 2005a), range size can be expected to be generally higher in poorly diversified lineages. However, this pattern remains to be tested.

Spatial/environmental effects on diversity skewness were observed for the Palaearctic but not for Nearctic 
flea assemblages. In general, higher skewness was characteristic of more northern assemblages occurring in colder environments. This parallels the pattern found for African primates reported by Heard \& Cox (2007), which they explained by the biogeography of primate radiation rather than by invoking environmental effects. In particular, they suggested that this gradient arose due to the southern distribution of Lorisidae, which is a sister clade to all other African primates. As a result, the occurrence of one lorisid species in northern localities is responsible for their high skewness values, whereas the occurrence of several lorisids in southern localities reduces skewness levels. We cannot distinguish any flea lineages that may have such an impact in the present study, so we believe that the observed gradient in the phylogenetic evenness of assemblage composition may be the outcome of environmental effects. One possible explanation may come from the higher intensity of diversification processes at lower latitudes due to higher energy input and the associated increase in mutation rate, acceleration of physiological processes, and shortened generation time (Rohde, 1992; Cardillo, Orme \& Owens, 2005). For example, the presence on the same host of multiple congeneric fleas belonging to various lineages (considered as an indicator of the rate of intra-host speciation) has been found more often in warmer than in colder regions (Krasnov et al., 2005b). It is possible that high energy input affects the rate of diversification of different flea lineages similarly, whereas, under low energy input, some lineages are still able to diversify and other lineages are more constrained. Consequently, some lineages may radiate or go extinct faster than other lineages directly because of external conditions (Krasnov et al., 2005b). The obvious result of this would be geographic variation in the diversity skewness of local assemblages.

By contrast, we did not detect any spatial gradient of diversity skewness for Nearctic assemblages. There can be at least two reasons for this lack of pattern. First, this can be attributed to differences in relief between the two land masses and the effect this may have had on their glacial history. Most of the mountain systems in the Nearctic consist of north-south oriented ridges, whereas there are many east-west oriented mountain systems in the Palaearctic. The latter have regularly created glacial refugia for a variety of species (Hewitt, 2004). This east-west fragmentation of the refugia could be one of the drivers of the latitudinal gradient in the diversity skewness of flea assemblages in the Palaearctic. Second, spatial patterns in diversity skewness can be a reflection of host-flea relationships rather than of the history of flea diversification per se. Indeed, our earlier study demonstrated the strong 'bottom-up' pattern of the relationship between host diversity and flea diversity in the Palaearctic but not in the Nearctic (Krasnov et al., 2007). From an ecological perspective, this suggested that host diversity controlled flea diversity in the Palaearctic, but not in the Nearctic. Furthermore, flea-host interactions in the Palaearctic appeared to be relatively specialized compared with those in the Nearctic because each flea species interacted with relatively fewer host species in the Palaearctic than in the Nearctic. Although spatial patterns of diversity skewness have not been studied for any mammalian taxon apart for primates, it is notable that in the latter this latitudinal gradient was seen in the Old World but not in the New World (Heard \& Cox, 2007). If such a gradient represents a general rule for mammals, then it is quite possible that spatial patterns in diversity skewness of parasites could be driven to a large extent by processes operating on their hosts. Further studies on the spatial patterns of diversity skewness in both hosts and their parasites are necessary for clarification of this issue.

\section{ACKNOWLEDGEMENTS}

We thank Brian Holmes for the kind permission to use data from his unpublished M. Sc. Thesis on fleas from Montana. This is publication no. 609 of the Mitrani Department of Desert Ecology and no. 247 of the Ramon Science Center.

\section{REFERENCES}

Agapow PM, Purvis A. 2002. Power of eight tree-shape statistics to detect nonrandom diversification: a comparison by simulation of two models of cladogenesis. Systematic Biology 51: 866-872.

Barraclough TG, Vogler A, Harvey PH. 1998. Revealing the factors that promote speciation. Philosophical Transactions of the Royal Society of London Series B, Biological Sciences 353: 241-249.

Blum MGB, François O. 2005. On statistical tests of phylogenetic tree imbalance: the Sackin and other indices. Mathematical Biosciences 195: 141-153.

Blum MGB, François O, Janson S. 2006. The mean, variance and joint distribution of two statistics sensitive to phylogenetic tree balance. Annals of Applied Probability 16: 2195-2214.

Cardillo M, Orme CDL, Owens IPF. 2005. Testing for latitudinal bias in diversification rates: an example using new world birds. Ecology 86: 2278-2287.

Cavalli-Sforza LL, Edwards AWF. 1967. Phylogenetic analysis: models and estimation procedures. Evolution 21: 550-570.

Chown SL. 1997. Speciation and rarity: separating cause from consequence. In: Kunin WE, Gaston KJ, eds. The biology of rarity: causes and consequences of rare-common differences. London: Chapman \& Hall, 91-109. 
Colless DH. 1982. Review of Phylogenetics: the theory and practice of phylogenetic systematics, by E. O. Wiley. Systematic Zoology 31: 100-104.

Dial KP, Marzluff JM. 1989. Nonrandom diversification within taxonomic assemblages. Systematic Zoology 38: 26-37.

Gaston KJ. 1998. Species-range size distributions: products of speciation, extinction and transformation. Philosophical Transactions of the Royal Society of London Series B, Biological Sciences 353: 219-230.

Gaston KJ. 2003. The structure and dynamics of geographic ranges. Oxford: Oxford University Press.

Harding EF. 1971. The probabilities of rooted treeshapes generated by random bifurcation. Advances in Applied Probability 3: 44-77.

Heard SB, Cox GH. 2007. The shapes of phylogenetic trees of clades, faunas, and local assemblages: exploring spatial pattern in differential diversification. The American Naturalist 169: E107-E118.

Hewitt GM. 2004. Genetic consequences of climatic oscillations in the Quaternary. Philosophical Transactions of the Royal Society of London Series B, Biological Sciences 359: 183-195.

Hickson RE, Slack KE, Lockhart P. 2000. Phylogeny recapitulates geography, or why New Zealand has so many species of skinks. Biological Journal of the Linnean Society 70: $415-433$.

Kirkpatrick M, Slatkin M. 1993. Searching for evolutionary patterns in the shape of a phylogenetic tree. Evolution 47: 1171-1181.

Krasnov BR, Poulin R, Shenbrot GI, Mouillot D, Khokhlova IS. 2005a. Host specificity and geographic range in haematophagous ectoparasites. Oikos 108: 449456.

Krasnov BR, Shenbrot GI, Khokhlova IS, Poulin R. 2005b. Diversification of ectoparasite assemblages and climate: an example with fleas parasitic on small mammals. Global Ecology and Biogeography 14: 167-175.

Krasnov BR, Shenbrot GI, Khokhlova IS, Poulin R. 2007. Geographic variation in the 'bottom-up' control of diversity: fleas and their small mammalian hosts. Global Ecology and Biogeography 16: 179-186.

Medvedev SG. 2005. An attempted system analysis of the evolution of the order of fleas (Siphonaptera). Lectures in memoriam N. A. Kholodkovsky, No. 57. Saint Petersburg: Russian Entomological Society and Zoological Institute of Russian Academy of Sciences (in Russian).

Medvedev SG. 2000a. Fauna and host-parasite associations of fleas (Siphonaptera) in different zoogeographical regions of the World. I. Entomological Review 80: 409-435.

Medvedev SG. 2000b. Fauna and host-parasite associations of fleas (Siphonaptera) in different zoogeographical regions of the World. II. Entomological Review 80: 640-655.

Medvedev SG, Krasnov BR. 2006. Fleas - permanent satellites of small mammals. In: Morand S, Krasnov BR, Poulin R, eds. Micromammals and macroparasites: from evolutionary ecology to management. Tokyo: SpringerVerlag, 161278.

Mooers AØ. 1995. Tree balance and tree completeness. Evolution 49: 379-384.

Mooers AØ, Heard SB. 1997. Inferring evolutionary process from phylogenetic tree shape. Quarterly Review of Biology 72: $31-54$.

Poulin R, Krasnov BR, Shenbrot GI, Mouillot D, Khokhlova IS. 2006. Evolution of host specificity in fleas: is it directional and irreversible? International Journal of Parasitology 36: 185-191.

Poulin R, Morand S. 2004. Parasite biodiversity. Washington, DC: Smithsonian Institution Press.

Rohde K. 1992. Latitudinal gradients in species diversity: the search for the primary cause. Oikos 65: 514-527.

Rosenzweig ML. 1995. Species diversity in space and time. Cambridge: Cambridge University Press.

Slowinski JB, Guyer C. 1989. Testing the stochasticity of patterns of organismal diversity: an improved null model. The American Naturalist 134: 907-921.

Slowinski JB, Guyer C. 1993. Testing whether certain traits have caused amplified diversification: an improved method based on a model of random speciation and extinction. The American Naturalist 142: 1019-1024.

Traub R. 1980. The zoogeography and evolution of some fleas, lice and mammals. In: Traub R, Starke H, eds. Fleas. Proceedings of the international conference on fleas, Ashton Wold, Peterborough, UK, 21-25 June 1977. Rotterdam: A. A. Balkema, 93-172.

Whiting MF, Whiting AS, Hastriter MW, Dittmar K. 2008. A molecular phylogeny of fleas (Insecta: Siphonaptera): origins and host associations. Cladistics 24: 677-707.

\section{SUPPORTING INFORMATION}

Additional Supporting Information may be found in the online version of this article:

Table S1. Data on mammalian and flea species richness in 41 regions.

Please note: Blackwell Publishing are not responsible for the content or functionality of any supporting materials supplied by the authors. Any queries (other than missing material) should be directed to the corresponding author for the article. 